\title{
Paranasal Sinus CT Is of Variable Value in Patients with Pediatric Cancer with Neutropenic Fever
}

W hen patients with pediatric cancer are found to have a fever without a source, CT of the paranasal sinuses, chest, abdomen, and pelvis has often been used as a screening tool. I question the role of sinus CT in this protocol.

It is commonly understood among radiologists that most children have some degree of paranasal sinus mucosal thickening, yet clinicians often use CT for answers when the result is almost always equivocal in the absence of symptomatology suggesting complicated sinus disease. ${ }^{1}$ To assess this, I looked at 19 children presenting to a desert-climate facility in southern Arizona for trauma without a history of sinusitis, and the average modified Lund-MacKay (mLM) score was 6.88 (95\% CI, 5.21-8.55). A same-site sample of patients with pediatric neutropenic fever assessed during the same period yielded a mean mLM of 4.67 (95\% CI, 3.38-5.96). A different sample of controls in northern Texas (humid subtropical climate) yielded an average mLM of 4.00 (95\% CI, 2.70-5.30). Simply put, mucosal thickening is a normal finding in children which likely depends to some degree on the environment in which they reside. While none of these children had signs or symptoms of complicated sinusitis, several of the pediatric cancer patients with neutropenic fever were subjected to specialty consultations based on the sinus findings, none of which resulted in additional therapy or continued concern for invasive fungal disease.

It is generally accepted that paranasal sinus CT is useful in

http://dx.doi.org/10.3174/ajnr.A6007 patients subject to hematopoietic stem cell transplantation, but the American College of Radiology Appropriateness Criteria assigns this a rating of $4,{ }^{2}$ the lower limit of "may be appropriate." The Children's Oncology Group position is to consider not routinely performing CT of the sinuses in patients without localizing signs or symptoms. ${ }^{3}$ My hope is that radiologists will do a better job of educating ordering providers regarding the utility of this test in this setting. Bedside sinus endoscopy is an effective alternative approach ${ }^{4}$ that offers the opportunity for sampling and avoids radiation.

\section{REFERENCES}

1. Tekes A, Palasis S, Durand DJ, et al; Expert Panel on Pediatric Imaging. ACR Appropriateness Criteria ${ }^{\circledR}$ Sinusitis-Child. J Am Coll Radiol 2018;15:S403-12 CrossRef Medline

2. Westra SJ, Karmazyn BK, Alazraki AL, et al; Expert Panel on Pediatric Imaging. ACR Appropriateness Criteria Fever Without Source or Unknown Origin-Child. J Am Coll Radiol 2016;13:922-30 CrossRef Medline

3. Children's Oncology Group. COG Supportive Care Endorsed Guidelines. January 31, 2019. https://www.childrensoncologygroup.org/ downloads/COG_SC_Guideline_Document.pdf. Accessed February 1,2019

4. Cohn SM, Rokala HR, Siegel JD, et al. Application of a standardized screening protocol for diagnosis of invasive mold infections in children with hematologic malignancies. Support Care Cancer 2016;24: 5025-33 CrossRef Medline
(1)C.M. Pfeifer Department of Radiology University of Texas Southwestern Medical Center Dallas, Texas 